\title{
Crack deflection in brittle media with heterogeneous interfaces and its application in shale fracking
}

\author{
Xiaguang Zeng, Yujie Wei* \\ LNM, Institute of Mechanics, Chinese Academy of Sciences, Beijing 100190, China
}

\section{A R T I C L E I N F O}

\section{Article history:}

Received 17 September 2016

Revised 24 November 2016

Accepted 31 December 2016

Available online 1 February 2017

\section{Keywords:}

Brittle materials

Shale hydraulic fracturing

Crack deflection

Strain energy release rate

Heterogeneous interfaces

\begin{abstract}
A B S T R A C T
Driven by the rapid progress in exploiting unconventional energy resources such as shale gas, there is growing interest in hydraulic fracture of brittle yet heterogeneous shales. In particular, how hydraulic cracks interact with natural weak zones in sedimentary rocks to form permeable cracking networks is of significance in engineering practice. Such a process is typically influenced by crack deflection, material anisotropy, crack-surface friction, crustal stresses, and so on. In this work, we extend the He-Hutchinson theory (He and Hutchinson, 1989) to give the closed-form formulae of the strain energy release rate of a hydraulic crack with arbitrary angles with respect to the crustal stress. The critical conditions in which the hydraulic crack deflects into weak interfaces and exhibits a dependence on crack-surface friction and crustal stress anisotropy are given in explicit formulae. We reveal analytically that, with increasing pressure, hydraulic fracture in shales may sequentially undergo friction locking, mode II fracture, and mixed mode fracture. Mode II fracture dominates the hydraulic fracturing process and the impinging angle between the hydraulic crack and the weak interface is the determining factor that accounts for crack deflection; the lower friction coefficient between cracked planes and the greater crustal stress difference favor hydraulic fracturing. In addition to shale fracking, the analytical solution of crack deflection could be used in failure analysis of other brittle media.
\end{abstract}

(c) 2017 Elsevier Ltd. All rights reserved.

\section{Introduction}

While the main stream of research on fracture is targeted at avoiding fracture, promoting fracture in a controllable way has gained attention in recent years owing to the interest in exploiting underground energy resources such as shale gas (Bažant et al., 2014). To achieve a high yield of shale gas collection, breakage of the low-permeability shale reservoirs where gas is encapsulated is desired. The development of hydraulic fracturing and horizontal well drilling techniques serves the purpose of cracking shales to form fracture networks in the gas reservoirs. Such a process may be influenced by crack deflection, anisotropy of shales, crack-plane friction, and crustal stress. As illustrated in Fig. 1, in addition to the hydraulic cracks, there are abundant material discontinuities in the shale stratum such as weak bedding interfaces and sealed natural fractures (Gale et al., 2014; Gale et al., 2007). A hydraulic fracture may cross these discontinuities, bend into them or be stopped by them. When the hydraulic fracture bends into and coalesces with the material discontinuities, a crack network starts to develop. Therefore, the interaction of a hydraulic crack with pre-existing weak regions and its deflection at the discontinuities are essential for the formation of such a network (Lee et al., 2015).

\footnotetext{
* Corresponding author.

E-mail address: yujie_wei@Inm.imech.ac.cn (Y. Wei).
} 


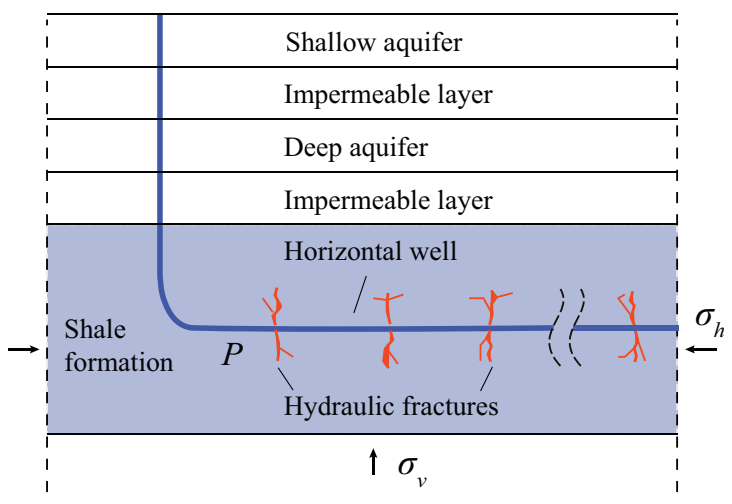

(a)

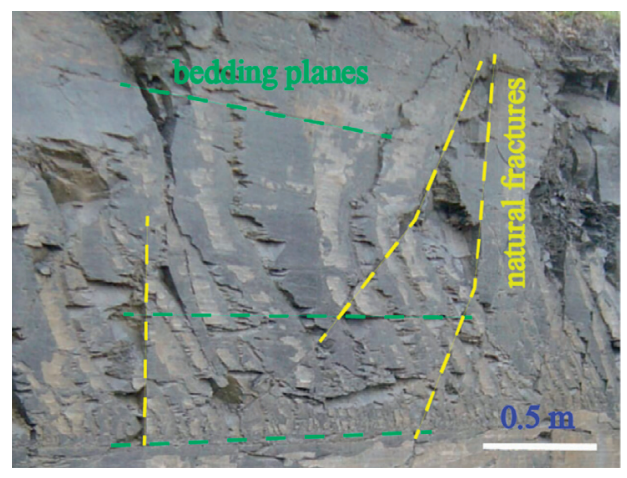

(b)

Fig. 1. The structure of shales and their surrounding environment. (a) Typical fracking process and the stress status of a shale stratum. The horizontal wellbore is in the direction of the minimum horizontal crustal stress $\sigma_{h}$; the hydraulic fractures would propagate across or along the weak regions under the influence of fluid pressure $P$, with crustal stresses $\sigma_{h}$ and $\sigma_{v}$. (b) Typical weak regions (material discontinuities) such as bedding planes and natural fractures in a shale outcrop (Gale et al., 2014).

Crack deflection or crack kink has been a subject of intensive research in the past. The core problems involve an accurate description of stress fields at the tip of an infinitesimal kinking crack and physically sound criteria to assess kink initiation and propagation (Williams, 1957; Erdogan and Sih, 1963; Bilby et al., 1977; Cotterell and Rice, 1980; Hayashi and Nemat-Nasser, 1981; Suresh and Shih, 1986; Amestoy and Leblond, 1992; Salvadori and Fantoni, 2016). When anisotropy emerges, the problem becomes extremely difficult to tackle. Zak and Williams (1963) gave the solution of symmetrical stress fields when a crack tip is perpendicular to a bi-material interface. For general anisotropy introduced by bi-material interfaces, Dundurs (1969) defined two parameters $D_{\alpha}=\frac{\mu_{1}\left(1-v_{2}\right)-\mu_{2}\left(1-v_{1}\right)}{\mu_{1}\left(1-v_{2}\right)+\mu_{2}\left(1-v_{1}\right)}$ and $D_{\beta}=\frac{\mu_{1}\left(1-2 \nu_{2}\right)-\mu_{2}\left(1-2 v_{1}\right)}{2\left[\mu_{1}\left(1-\nu_{2}\right)+\mu_{2}\left(1-v_{1}\right)\right]}$, which are important to quantify the elastic mismatch by two isotropic materials. Here, $\mu_{1}$ and $\mu_{2}$ denote the shear moduli and $v_{1}$ and $v_{2}$ denote Poisson's ratios of the materials 1 and 2. Cook and Erdogan (1972) solved the elasticity of the perpendicular crack-interface problem in terms of the two Dundurs' numbers in an integral form and investigated the crack path problem numerically by adopting the maximum stress criterion for crack propagation. In parallel, both a stress intensity factor criterion (Irwin, 1957) and a strain energy release rate criterion (Griffith, 1921) have been employed to forecast the propagation of a crack perpendicular or parallel to a bi-material interface, including works by Lu and Erdogan (1983), Hutchinson et al. (1987), and Sun and Jih (1987), among others. He and Hutchinson (1989) solved the elastic problem of crack deflection in two dissimilar isotropic materials. They considered an oblique interface between two different isotropic media and gave the critical conditions in which the primary crack deflects into the interface. The authors supplied numerical results of the integral-form solutions to predict the crack extension paths for typical crack-interface orientations. Later on, Gupta et al. (1992), Martínez and Gupta (1994) applied the same strategy to examine crack deflection in two orthotropic media.

By contrast, boosted by the rapid development in computational power and numerical techniques, numerous efforts from the numerical side have been carried out to investigate crack-deflection problems. For example, Mi et al. (1998) developed a method incorporating interface elements within the non-linear finite element method (FEM) and successfully simulated the mixed mode delamination of fiber-composites. Parmigiani and Thouless (2006) studied crack deflection and crack penetration using a cohesive model that incorporated both strength and fracture toughness simultaneously. They showed that the cohesive model is capable of simulating the deflection-penetration competition behavior. Wei et al. (2009) modeled the crack propagation across twist-misoriented grain boundaries in lamellar microstructures using a dynamic FEM and investigated its influence on the fracture toughness of such heterogeneous solids.

However, the hydraulic fracture problem is more complicated considering the highly anisotropic nature of shales. Factors such as pre-existing cracks, bedding planes, frictional effect, crustal stresses, and liquid-solid interaction may collectively influence the propagation of a hydraulic crack (Warpinski and Teufel, 1987; Zhang and Jeffrey, 2006; Bunger and Detournay, 2008; Chuprakov et al., 2014). Such complexity renders it difficult to carry out theoretical analysis, and numerical methods were broadly employed to examine the hydraulic fracture: Zhang et al. (2007), Taleghani and Olson (2011, 2014), Weng et al. (2011), Kresse et al. (2013), Guo et al. (2015) and Zou et al. (2016) simulated the interaction between a hydraulic fracture and frictional or sealed natural cracks. A coupled, implicit time-stepping numerical model was developed by Bunger et al. (2013) to capture the hydraulic fracture that grows near a free surface and curves towards the surface. Combined with very recent developments in numerical models for the initiation and growth of hydraulic fractures by Lecampion and Desroches (2015) and Grassl et al. (2015), we learned that the elastic anisotropy, shale strength and toughness, weak zone strength and toughness, crack-plane friction, and impinging angle between the hydraulic fracture and natural cracks play critical roles in the formation of fracture networks with high permeability. Notably, a hydraulic crack is of finite length and its impinging angle is also well defined in all numerical models. Hence, conclusions drawn from such calculations are difficult 


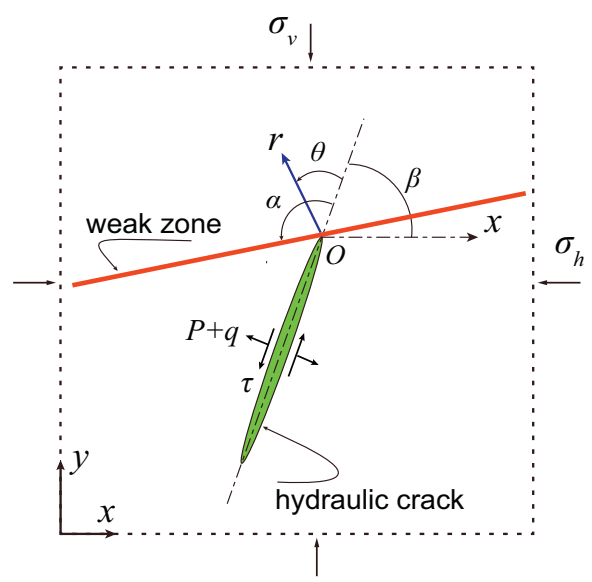

(a)

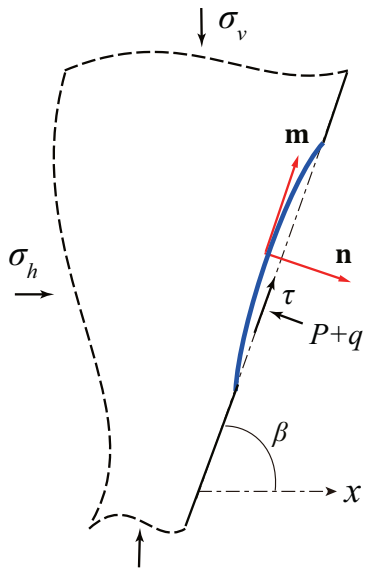

(b)

Fig. 2. Geometric information for a hydraulic crack interacting with a pre-existing weak interface. (a) Definition of the hydraulic crack and the weak interface, where $0 \leq \beta \leq \pi / 2,-\pi<\theta<\pi$ and $-\pi<\alpha<\pi$. (b) A free-body diagram to show the force equilibrium in the crack surface: the traction exerted on the surface is $-q \mathbf{n}-\tau \mathbf{m}$, and the hydraulic pressure on the surface is $-P \mathbf{n}$. Here $(\mathbf{n}, \mathbf{m})$ defines a local coordinate with $\mathbf{n}$ denoting the crack surface normal and $\mathbf{m}$ denoting the surface tangential.

to generalize. In this paper, we seek to supply an analytical framework for crack deflection in one type of brittle material with heterogeneous interfaces. By extending the energy-based crack deflection criterion in the He-Hutchinson theory (1989), we supply closed-form solutions for the shale hydraulic crack deflection under the influence of crack-plane friction and crustal stress. Such solutions could be employed in engineering practice to estimate the critical conditions for successful hydraulic fracturing, given that we know the approximate crustal stress and frictional behavior of the shale stratum.

\section{Description of the problem}

Shales are fine-grained, sedimentary rocks with a complex composition that are characterized by breaking along their weak bedding or layering planes. Such natural weak zones have a great influence on the mechanical properties of shales (Zeng and Wei, 2016). Commonly, shales are regarded as brittle and transversely isotropic materials, with a symmetry axis perpendicular to the sedimentary planes (Vasin et al., 2013). For simplicity, but without loss of physics, we consider the bedding interfaces and natural fractures as material discontinuities that have no thickness and different fracture toughness values than that of the shale matrix. To supply analytical solutions to hydraulic fracture in shales under the influence of crack-plane friction and crustal stress, we make the following assumptions:

(1) The shale under consideration is an isotropic and brittle medium (Chuprakov et al., 2014). Therefore, linear elastic fracture mechanics is applicable.

(2) The anisotropic nature of shales is represented by distinct fracture toughness in weak regions and the matrix. Such material discontinuity across the weak regions plays a central role in determining the crack propagation direction.

(3) Pumping fluid leads to a uniform pressure on the hydraulic fracture surfaces. We also neglect the so-called lag zone (Bunger and Detournay, 2008) during the extension of a hydraulic crack.

Based on these assumptions, we consider a representative shale body with a central crack of length $2 c$, as illustrated in Fig. 2a. The angle between the $\mathrm{x}$-axis and the crack plane is $\beta$, with $0 \leq \beta \leq \pi / 2$. A hydraulic pressure $P$ is applied in the crack. The contact pressure between the two crack planes is $q$ and their frictional shear is $\tau$. The shale is subjected to crustal stresses $\sigma_{h}$ and $\sigma_{v}$ in the horizontal (x-axis) and the vertical (y-axis) directions, respectively. The matrix has a Young's modulus $E$ and a Poisson's ratio $\nu$. The fracture toughness of the matrix is $G_{m c}$ whereas that in the weak interface is $G_{d c}$. We let $G_{\theta}$ denote the strain energy release rate as the hydraulic crack extends in the $\theta$ direction, which is positive when rotating counter-clockwise from the crack extension line to the kinking direction, as shown in Fig. 2a. We let $\alpha$ denote the angle from the crack extension line to the weak interface, which is positive for counter-clockwise rotation as well.

\section{Theoretical analysis}

\subsection{Crack deflection criterion}

For the crack shown in Fig. 2a, we sought to predict the crack propagation path. To achieve this goal, we should have a physically sound fracture criterion to determine when and how the crack kink occurs. Several types of criteria are broadly 
employed for isotropic brittle failure, such as the maximum principal stress-based criterion (Erdogan and Sih, 1963) and that based on the maximum energy release rate (Hussain et al., 1973; Nuismer, 1975). Predictions drawn from these types of criteria agree reasonably well with experimental observations of isotropic brittle failure. Following the maximum energy release rate criterion, He and Hutchinson (1989) developed a crack deflection criterion based on the ratio of energy release rates. Such a criterion is particularly useful in shales with anisotropic fracture toughness due to the presence of natural weak planes, as demonstrated by Taleghani and Olson $(2011,2014)$ in their numerical simulations for shale fracking. In this paper, we define the crack propagation along a weak interface as the crack deflection and the propagation in the direction of maximum energy release rate as the isotropic extension. Following the energy-based criterion used by He and Hutchinson (1989), crack deflection would occur if the following two conditions are satisfied (otherwise, the crack isotropic extension would happen when $G_{\theta \max }=G_{m c}$ ):

Firstly, the strain energy release rate $G_{\alpha}$ when the crack extends along the weak region should meet

$$
G_{\alpha}=G_{d c},
$$

and secondly,

$$
\frac{G_{\alpha}}{G_{\theta \max }}>\frac{G_{d c}}{G_{m c}}
$$

where $G_{\theta \max }$ is the maximum strain energy release rate when the crack extends in the matrix and $G_{d c}$ and $G_{m c}$ represent the Mode I fracture toughness of the weak region and the matrix, respectively. Hence, calculating the strain energy release rate at an arbitrary angle $\theta$ is required before we may apply the criteria given in Eqs. (1) and (2).

The strain energy release rate for a deflected crack with a zero kink length at an angle $\theta$ under mixed mode loading was given by Nuismer (1975) as:

$$
G_{\theta}=\left(K_{1 \theta}^{2}+K_{2 \theta}^{2}\right) / E^{\prime}
$$

where $K_{1 \theta}=\frac{1}{2} \cos \left(\frac{\theta}{2}\right)\left[K_{I}(1+\cos \theta)-3 K_{I I} \sin \theta\right]$ and $K_{2 \theta}=\frac{1}{2} \cos \left(\frac{\theta}{2}\right)\left\{K_{I} \sin \theta+K_{I I}[3 \cos \theta-1]\right\}$ for $K_{I}$ and $K_{I I}$, the Mode I and Mode II stress intensity factors of the primary crack, respectively, and $E^{\prime}=E$ for the stress plane and $E^{\prime}=E /\left(1-v^{2}\right)$ for the strain plane. It is noted that Eq. (3) is approximate. A thorough discussion and corresponding numerical calculations have been made by Hutchinson and Suo (1992) for more general cases if the kinked crack is of finite length. In that circumstance, the ratio of the energy release rate of the primary crack when is advances straight-ahead to that of the kinked crack is of the following form (Hutchinson and Suo, 1992),

$$
G_{0} / G_{\theta}=F(\theta, \psi, \eta)
$$

where $\psi=\operatorname{atan}\left(K_{I I} / K_{I}\right)$ is the measure of mode II to mode I loading acting on the main crack, called phase angle of loading, $\eta$ is the in-plane stress parameter. In what follows, we limit our discussion for the initiation of crack kink. Hence the kink crack has zero-length. To obtain the explicit expression of Eq. (3), we notice that the circumferential stress is related to $K_{1 \theta}$ via $\sigma_{\theta \theta}=K_{1 \theta} / \sqrt{2 \pi r}$ and the shear stress component $\tau_{r \theta}=K_{2 \theta} / \sqrt{2 \pi r}$, where $r$ is the radial coordinate of the local polar coordinate system shown in Fig. 2a. When $K_{1 \theta}<0, \sigma_{\theta \theta}$ is compressive and does not contribute to the energy leading to the extension of the crack. We exclude it from the strain energy release rate and rewrite Eq. (3) as:

$$
G_{\theta}=\left\{\begin{array}{l}
K_{2 \theta}^{2} / E^{\prime}, K_{1 \theta}<0 \\
\left(K_{1 \theta}^{2}+K_{2 \theta}^{2}\right) / E^{\prime}, K_{1 \theta} \geq 0
\end{array}\right.
$$

for $-\pi<\theta<\pi$. By substituting the expressions for $K_{1 \theta}$ and $K_{2 \theta}$ into Eq. (5), we obtain the K-type formula for the strain energy release rate:

$$
G_{\theta}=\left\{\begin{array}{lr}
\frac{\cos ^{2}\left(\frac{\theta}{2}\right)\left[K_{I I}-3 K_{I I} \cos \theta-K_{I} \sin \theta\right]^{2}}{4 E^{\prime}}, & \text { if } K_{1 \theta}<0 \\
\frac{\cos ^{2}\left(\frac{\theta}{2}\right)\left[K_{I}^{2}+5 K_{I I}^{2}+\left(K_{I}^{2}-3 K_{I I}^{2}\right) \cos \theta-4 K_{I} K_{I I} \sin \theta\right]}{2 E^{\prime}}, & \text { when } K_{1 \theta} \geq 0
\end{array} .\right.
$$

With Eq. (6), we deduce the maximum strain energy release rate as:

$$
G_{\theta \max }=\frac{\cos ^{2}\left(\frac{\theta_{0}}{2}\right)\left[K_{I}^{2}+5 K_{I I}^{2}+\left(K_{I}^{2}-3 K_{I I}^{2}\right) \cos \theta_{0}-4 K_{I} K_{I I} \sin \theta_{0}\right]}{2 E^{\prime}} .
$$

The angle $\theta_{0}$ along which $G_{\theta}$ maximizes is determined by solving the equation: $K_{I}^{2} \sin \theta_{0}\left[1+\cos \theta_{0}\right]-2 K_{I} K_{I I}$ $\left[\sin ^{2} \theta_{0}-\cos ^{2} \theta_{0}-\cos \theta_{0}\right]+K_{I I}^{2} \sin \theta_{0}\left[1-3 \cos \theta_{0}\right]=0$. The solution is given as:

$$
\theta_{0}=\left\{\begin{array}{l}
-\operatorname{acos}\left(\frac{3 K_{I I}^{2}+\sqrt{K_{I}^{4}+8 K_{I}^{2} K_{I I}^{2}}}{K_{I}^{2}+9 K_{I I}^{2}}\right), \quad \text { for } K_{I}>0 \text { and } K_{I I}>0 \\
\operatorname{acos}\left(\frac{3 K_{I}^{2}+\sqrt{K_{I}^{4}+8 K_{I}^{2} K_{I I}^{2}}}{K_{I}^{2}+9 K_{I I}^{2}}\right), \quad \text { for } K_{I}>0 \text { and } K_{I I} \leq 0 \\
\pm \operatorname{acos}(1 / 3), \quad \text { when } K_{I}=0 \text { and } K_{I I} \neq 0
\end{array}\right.
$$


By substituting Eq. (8) into (7), we have:

$$
\begin{aligned}
& G_{\theta \max }=\frac{\left(\bar{K}_{a}^{2}+\bar{K}_{b}^{2}\right)\left[K_{I}^{2}+4 K_{I} K_{I I} \sqrt{1-\frac{\bar{K}_{a}^{4}}{\bar{K}_{b}^{4}}}+\frac{\left(K_{I}^{2}-3 K_{I I}^{2}\right) \bar{K}_{a}^{2}}{\bar{K}_{b}^{2}}+5 K_{I I}^{2}\right]}{4 E^{\prime} \bar{K}_{b}^{2}}, \text { for } K_{I}>0 \text { and } K_{I I}>0, \\
& G_{\theta \max }=\frac{\left(\bar{K}_{a}^{2}+\bar{K}_{b}^{2}\right)\left[K_{I}^{2}-4 K_{I} K_{I I} \sqrt{1-\frac{\bar{K}_{a}^{4}}{\bar{K}_{b}^{4}}}+\frac{\left(K_{I}^{2}-3 K_{I I}^{2}\right) \bar{K}_{a}^{2}}{\bar{K}_{b}^{2}}+5 K_{I I}^{2}\right]}{4 E^{\prime} \bar{K}_{b}^{2}}, \text { for } K_{I}>0 \text { and } K_{I I} \leq 0, \\
& G_{\theta \max }=\frac{4 K_{I I}^{2}}{3 E^{\prime}}, \text { if } K_{I}=0 \text { and } K_{I I} \neq 0,
\end{aligned}
$$

where $\bar{K}_{a}^{2}=\sqrt{K_{I}^{4}+8 K_{I}^{2} K_{I I}^{2}}+3 K_{I I}^{2}$ and $\bar{K}_{b}^{2}=K_{I}^{2}+9 K_{I I}^{2}$. The strain energy release rate can alternatively be expressed in terms of J-integrals (Rice, 1968), as detailed in Appendix A.

We may also express Eq. (8) and the ratio in terms of the phase angle. Consider the special case of zero-kink length, we have (Hutchinson and Suo, 1992),

$$
G_{0} / G_{\theta}=F(\theta, \psi, 0),
$$

Using Eq. (6) and the definition of $\psi$, we obtain an expression of the ratio for the zero-kinked case:

$$
G_{0} / G_{\theta}=\left\{\begin{array}{ll}
\frac{8\left(1+\tan ^{2} \psi\right)}{(1+\cos \theta)(\tan \psi-3 \cos \theta \tan \psi-\sin \theta)^{2}}, & \text { if } \tan \psi \tan \left(\frac{\theta}{2}\right)>0 \\
\frac{4}{(1+\cos \theta)(2 \cos (2 \psi+\theta)-2 \cos (2 \psi)-\cos \theta+3)}, & \text { when } \tan \psi \tan \left(\frac{\theta}{2}\right) \leq 0
\end{array} .\right.
$$

Hence the ratio of the straight-ahead energy release rate of the main crack to the maximum release rate is:

$$
G_{0} / G_{\theta \max }=\frac{4}{\left(1+\cos \theta_{0}\right)\left(2 \cos \left(2 \psi+\theta_{0}\right)-2 \cos (2 \psi)-\cos \theta_{0}+3\right)},
$$

the corresponding kinking angle $\theta_{0}$ is determined as

$$
\theta_{0}=\left\{\begin{array}{cl}
-\operatorname{acos}\left(\frac{3 \tan ^{2} \psi+\sqrt{1+8 \tan ^{2} \psi}}{1+9 \tan ^{2} \psi}\right), & \text { for } 0<\psi<\pi / 2 \\
\operatorname{acos}\left(\frac{3 \tan ^{2} \psi+\sqrt{1+8 \tan ^{2} \psi}}{1+9 \tan ^{2} \psi}\right), & \text { for }-\pi / 2<\psi \leq 0 \\
\pm \operatorname{acos}(1 / 3), & \text { when } \psi=\mp \pi / 2
\end{array} .\right.
$$

\subsection{Criterion application in the fracking problem}

With the modified definition of the strain energy release rate given in Eq. (6) and its maximum shown in Eq. (9), we apply the theory to hydraulic fracture deflection problems. Employing the cracking criterion in Eqs. (1) and (2), we sought to obtain the analytical solutions for crack deflection during shale fracking.

\subsubsection{Determining the stress intensity factors}

For the stressed crack shown in Fig. 2a, the surface contact pressure $q$ and friction $\tau$ depend on the fluid pressure $P$. When $P>\sigma_{h} \sin ^{2}(\beta)+\sigma_{v} \cos ^{2}(\beta)$, the crack is open. Therefore, $q=0$ and $\tau=0$. Conversely, for $P \leq \sigma_{h} \sin { }^{2}(\beta)+$ $\sigma_{v} \cos ^{2}(\beta)$, the crack is closed and the crack surfaces are subjected to frictional resistance and contact pressure. We take a free-body that includes one side of the crack to relate $q$ and $\tau$ with the fluid pressure and crustal stresses, as shown in Fig. 2 b. The crack surface traction can be characterized by the two scalar components $(-q,-\tau)$ in the local coordinate defined by the surface normal $\mathbf{n}$ and its tangential $\mathbf{m}$. For simplicity, we assume $\sigma_{v}<\sigma_{h}$ unless stated otherwise. According to Coulomb's friction law, the stress statuses in the crack surface may fall into the following scenarios:

I. If $|\tau| \leq f|q|$, no slip occurs and we obtain $q$ and $\tau$ for the force equilibrium in the normal and tangential directions, respectively:

$$
\begin{aligned}
& q=\sigma_{h} \sin ^{2} \beta+\sigma_{v} \cos ^{2} \beta-P, \\
& \tau=\left(\sigma_{v}-\sigma_{h}\right) \sin \beta \cos \beta .
\end{aligned}
$$

II. If $\left(\sigma_{h}-\sigma_{v}\right) \sin \beta \cos \beta>f|q|$, the body is on the verge of slipping along $\mathbf{m}$ and

$$
\tau=-f\left[\sigma_{h} \sin ^{2} \beta+\sigma_{v} \cos ^{2} \beta-P\right] .
$$

The equilibrium condition $q=\sigma_{h} \sin ^{2} \beta+\sigma_{v} \cos ^{2} \beta-P$ still holds. 

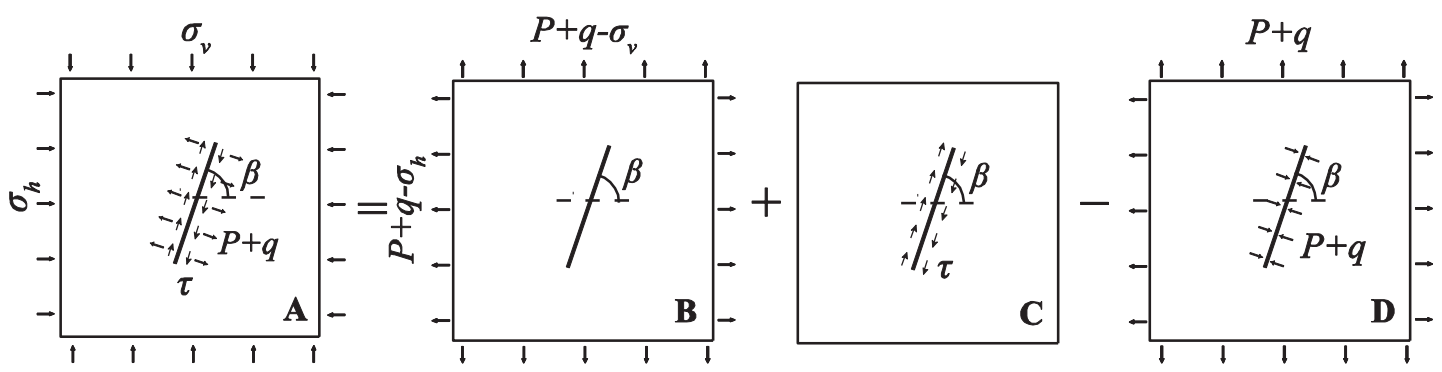

Fig. 3. Superposition of the stress intensity factor $K$ for the fracking problem. We see that the stress intensity factor $K_{\mathrm{A}}$ in ' $A$ ' is connected with other factors via $K_{\mathrm{A}}=K_{\mathrm{B}}+K_{\mathrm{C}}-K_{\mathrm{D}}$.

III. If $\left(\sigma_{v}-\sigma_{h}\right) \sin \beta \cos \beta>f|q|$, the body is on the verge of slipping against $\mathbf{m}$; thus, we obtain

$$
\tau=f\left[\sigma_{h} \sin ^{2} \beta+\sigma_{v} \cos ^{2} \beta-P\right],
$$

and, according to the force balance, we have $q=\sigma_{h} \sin ^{2} \beta+\sigma_{v} \cos ^{2} \beta-P$.

To deduce the stress intensity factors of the fracking problem subjected to the loading conditions shown in Fig. 2a, we decompose the stress boundaries into multiple statuses, as shown in Fig. 3. Thus, we may write out the corresponding stress intensity factors for each simplified status for which a theoretical solution is available. By employing the superposition principle, we then obtain the stress intensity factor $K$ of the original fracking problem, i.e., $K_{\mathrm{A}}=K_{\mathrm{B}}+K_{\mathrm{C}}-K_{\mathrm{D}}$. The sample in graph 'D' in Fig. 3 is loaded with remote tensile loading $P+q$ and crack closing loading $P+q$. In such circumstances, the crack is closed and $K_{\mathrm{D}}=0$. Thus, we have $K_{\mathrm{A}}=K_{\mathrm{B}}+K_{\mathrm{C}}$. Using the formulae for a slanted central crack of length $2 c$ in a biaxial stress status (Anderson, 2005), the stress intensity factors for the sample in graph ' $\mathrm{B}$ ' in Fig. 3 is given as:

$$
\begin{aligned}
& K_{\mathrm{BI}}=\left[\left(P+q-\sigma_{h}\right) \sin ^{2} \beta+\left(P+q-\sigma_{v}\right) \cos ^{2} \beta\right] \sqrt{\pi c}, \\
& K_{\mathrm{BII}}=\left(\sigma_{h}-\sigma_{v}\right) \sin \beta \cos \beta \sqrt{\pi c},
\end{aligned}
$$

for $K_{\mathrm{BI}}$ and $K_{\mathrm{BII}}$, the Mode I and Mode II cases, respectively. The stress intensity factors $K_{\mathrm{CI}}$ and $K_{\mathrm{CII}}$ for Mode I and II in ' $\mathrm{C}$ ' of Fig. 3 can be obtained using the formulae given by Tada et al. (2000):

$$
K_{C I}=0, \quad K_{\mathrm{CII}}=\tau \sqrt{\pi c} .
$$

Hence, the stress intensity factors of the fracking model in 'A' of Fig. 3 are given as:

$$
\begin{aligned}
& K_{I}=K_{\mathrm{BI}}+K_{\mathrm{CI}}=\left[\left(P+q-\sigma_{h}\right) \sin ^{2} \beta+\left(P+q-\sigma_{v}\right) \cos ^{2} \beta\right] \sqrt{\pi c}, \\
& K_{I I}=K_{\mathrm{BII}}+K_{\mathrm{CII}}=\left[\left(\sigma_{h}-\sigma_{v}\right) \sin \beta \cos \beta+\tau\right] \sqrt{\pi c} .
\end{aligned}
$$

Because $\sigma_{v}<\sigma_{h}$, the third scenario in the discussion for $q$ and $\tau$, that the free-body is on the verge of slipping against $\mathbf{m}$, would never occur. We can now substitute expressions of $q$ and $\tau$ for the scenarios I and II into Eq. (17) and obtain the stress intensity factors for the aforementioned circumstances for the fracking problem:

Case I: $\sigma_{h} \sin ^{2} \beta+\sigma_{v} \cos ^{2} \beta-P \geq 0$ and $\left|\left(\sigma_{h}-\sigma_{v}\right) \sin \beta \cos \beta\right| \leq f\left[\sigma_{h} \sin ^{2} \beta+\sigma_{v} \cos ^{2} \beta-P\right]$. Hence, we have

$$
K_{I}=0, K_{I I}=0
$$

Case II: $\sigma_{h} \sin ^{2} \beta+\sigma_{v} \cos ^{2} \beta-P>0$ and $\left(\sigma_{h}-\sigma_{v}\right) \sin \beta \cos \beta>f\left[\sigma_{h} \sin ^{2} \beta+\sigma_{v} \cos ^{2} \beta-P\right]$. Thus, we have

$$
K_{I}=0, K_{I I}=\left\{\left(\sigma_{h}-\sigma_{v}\right) \sin \beta \cos \beta+f\left[P-\sigma_{h} \sin ^{2} \beta-\sigma_{v} \cos ^{2} \beta\right]\right\} \sqrt{\pi c} .
$$

Case III: $\sigma_{h} \sin ^{2} \beta+\sigma_{v} \cos ^{2} \beta-P<0$. The stress intensity factors are given as:

$$
K_{I}=\left[P-\sigma_{h} \sin ^{2} \beta-\sigma_{v} \cos ^{2} \beta\right] \sqrt{\pi c}, K_{I I}=\left(\sigma_{h}-\sigma_{v}\right) \sin \beta \cos \beta \sqrt{\pi c} .
$$

With known stress intensity factors, the strain energy release rate could be explicitly formulated. We need to determine when a hydraulic crack deflects, which is shown below.

\subsubsection{Hydraulic fracture deflection conditions}

For the primary crack to kink and extend along the weak interface, Eq. (1), i.e., $G_{\alpha}=G_{d c}$, should be satisfied. By substituting the stress intensity factors from Eq. (18) into (6), we obtain the strain energy release rate along the interface. We notice 
that $G_{\alpha}=0$ for Case I and the solution is trivial. Let $\bar{s}_{q}=\sigma_{h} \sin ^{2} \beta+\sigma_{v} \cos ^{2} \beta-P$ and $\bar{s}_{\tau}=\left(\sigma_{h}-\sigma_{v}\right) \sin \beta$ cos $\beta$. For Case II, we have $\bar{s}_{q}>0$ and $\bar{s}_{\tau}>f \bar{s}_{q}$, hence:

$$
G_{\alpha}=\left\{\begin{array}{cl}
\frac{\pi c \cos ^{2}\left(\frac{\alpha}{2}\right)[1-3 \cos \alpha]^{2}\left(\bar{s}_{\tau}-f \bar{s}_{q}\right)^{2}}{4 E^{\prime}}, & \text { when } \sin \alpha>0 \\
\frac{\pi c \cos ^{2}\left(\frac{\alpha}{2}\right)[5-3 \cos \alpha]\left(\bar{s}_{\tau}-f \bar{s}_{q}\right)^{2}}{2 E^{\prime}}, & \text { if } \sin \alpha \leq 0
\end{array} .\right.
$$

For Case III, $\bar{s}_{q}<0$, we have:

$$
G_{\alpha}=\left\{\begin{array}{c}
\frac{\pi c \cos ^{2}\left(\frac{\alpha}{2}\right)\left\{[6 \cos \alpha-2] \bar{s}_{\tau}-2 \bar{s}_{q} \sin \alpha\right\}^{2}}{16 E^{\prime}}, \text { if }(1+\cos \alpha) \bar{s}_{q}+3 \sin \alpha \bar{s}_{\tau}>0 \\
\frac{\pi c \cos ^{2}\left(\frac{\alpha}{2}\right)\left\{4 \sin \alpha \bar{s}_{\tau} \bar{s}_{q}+(1+\cos \alpha) \bar{s}_{q}^{2}+(5-3 \cos \alpha) \bar{s}_{\tau}^{2}\right\}}{2 E^{\prime}}, \text { if }(1+\cos \alpha) \bar{s}_{q}+3 \sin \alpha \bar{s}_{\tau} \leq 0
\end{array} .\right.
$$

Next, we could obtain the maximum strain energy release rate for Case II by submitting Eq. (18) into (9),

$$
G_{\theta \max }=\frac{4 \pi c\left(\bar{s}_{\tau}-f \bar{s}_{q}\right)^{2}}{3 E^{\prime}} .
$$

The corresponding angles are:

$$
\theta_{0}= \pm \operatorname{acos}(1 / 3)
$$

For Case III, we submit Eq. (18) into (7). Hence, $G_{\theta \max }$ is written as:

$$
G_{\theta \max }=\frac{\pi \cos ^{2}\left(\frac{\theta_{0}}{2}\right)\left\{4 \bar{s}_{\tau} \bar{s}_{q} \sin \theta_{0}+\left[1+\cos \theta_{0}\right] \bar{s}_{q}^{2}+\left[5-3 \cos \theta_{0}\right] \bar{s}_{\tau}^{2}\right\}}{2 E^{\prime}} .
$$

For the general case when $0<\beta<\pi / 2, \theta_{0}$ is determined as:

$$
\theta_{0}^{ \pm}= \pm \operatorname{acos}\left(\frac{3 \bar{s}_{\tau}^{2}+\sqrt{\bar{s}_{q}^{2}\left(\bar{s}_{q}^{2}+8 \bar{s}_{\tau}^{2}\right)}}{\bar{s}_{q}^{2}+9 \bar{s}_{\tau}^{2}}\right)
$$

When $\sigma_{h}<P$, or $\sigma_{h} \geq P$ and $\sigma_{v}<\sec ^{2} \beta\left(P-\sigma_{h} \sin ^{2} \beta\right), \theta_{0}=\theta_{0}^{-}$. If $\sigma_{h}<P$ and $\sigma_{v} \leq \sec ^{2} \beta\left(P-\sigma_{h} \sin ^{2} \beta\right)$, or $\sigma_{h}=P$ and $\sigma_{v}=\sec ^{2} \beta\left(P-\sigma_{h} \sin ^{2} \beta\right)$, we have $\theta_{0}=\theta_{0}^{+}$. For the special case when $\beta=0$ and $\sigma_{v} \leq P$, or $\beta=\pi / 2$ and $\sigma_{h} \leq P$, $\theta_{0}=\theta_{0}^{+}$. By knowing $G_{\alpha}$ and $G_{\theta \max }$, we examine whether the hydraulic fracture can deflect into the weak interface. Substituting Eq. (19) and (20a) or (21a) into Eq. (2), we obtain the following conditions for hydraulic fracture deflection:

For Case II, we have $\bar{s}_{q}>0$ and $\bar{s}_{\tau}>f \bar{s}_{q}$ and the deflection criterion requires:

$$
\frac{G_{d c}}{G_{m c}}<\frac{G_{\alpha}}{G_{\theta \max }}=\left\{\begin{array}{cl}
3 \cos ^{2}\left(\frac{\alpha}{2}\right)[1-3 \cos \alpha]^{2} / 16, & \text { when } \sin \alpha>0 \\
3 \cos ^{2}\left(\frac{\alpha}{2}\right)[5-3 \cos \alpha] / 8, & \text { if } \sin \alpha \leq 0
\end{array} .\right.
$$

For Case III, $\bar{s}_{q}<0$, we have:

$$
\frac{G_{d c}}{G_{m c}}<\frac{G_{\alpha}}{G_{\theta \max }}=\frac{\sec ^{2}\left(\frac{\theta_{0}}{2}\right) \cos ^{2}\left(\frac{\alpha}{2}\right)\left\{[6 \cos \alpha-2] \bar{s}_{\tau}-2 \sin \alpha \bar{s}_{q}\right\}^{2}}{8\left\{4 \sin \theta_{0} \bar{s}_{\tau} \bar{s}_{q}+\left[1+\cos \theta_{0}\right] \bar{s}_{q}^{2}+\left[5-3 \cos \theta_{0}\right] \bar{s}_{\tau}^{2}\right\}} .
$$

if $[1+\cos (\alpha)] \bar{s}_{q}+3 \sin (\alpha) \bar{s}_{\tau}>0$. When $[1+\cos \alpha] \bar{s}_{q}+3 \sin \alpha \bar{s}_{\tau} \leq 0$, the criterion is given as:

$$
\frac{G_{d c}}{G_{m c}}<\frac{G_{\alpha}}{G_{\theta \max }}=\frac{\sec ^{2}\left(\frac{\theta_{0}}{2}\right) \cos ^{2}\left(\frac{\alpha}{2}\right)\left\{4 \sin \alpha \bar{s}_{\tau} \bar{s}_{q}+[\cos \alpha+1] \bar{s}_{q}^{2}+[5-3 \cos \alpha] \bar{s}_{\tau}^{2}\right\}}{4 \bar{s}_{\tau} \bar{s}_{q} \sin \theta_{0}+\left[\cos \theta_{0}+1\right] \bar{s}_{q}^{2}+\left[5-3 \cos \theta_{0}\right] \bar{s}_{\tau}^{2}} .
$$

Here, $\theta_{0}$ is given by Eq. (21b). For the special case of symmetry loading, $\beta=\pi / 2$, we have the simple relations for crack deflection criterion as:

$$
G_{d c}=G_{\alpha}=\frac{\pi \cos ^{4}\left(\frac{\alpha}{2}\right)\left(P-\sigma_{h}\right)^{2}}{E^{\prime}}
$$

and

$$
\frac{G_{d c}}{G_{m c}}<\frac{G_{\alpha}}{G_{\theta \max }}=\cos ^{4}\left(\frac{\alpha}{2}\right) .
$$

Notably, for the other special case when $\beta=0, \sigma_{h}$ in Eq. (23a) should be replaced by $\sigma_{v}$. 


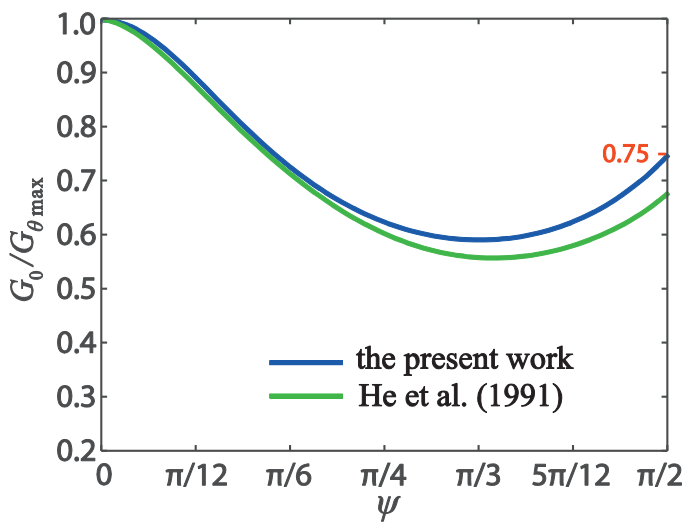

(a)

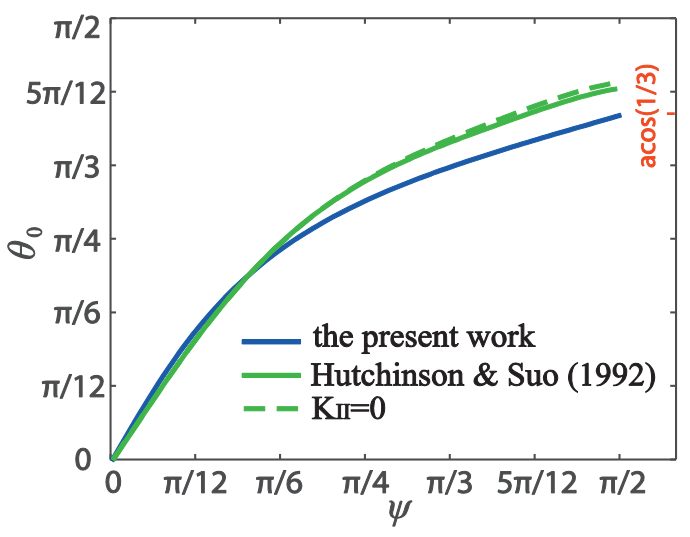

(b)

Fig. 4. Evaluation on the approximation of the Nuismer's equation for crack kinking analysis. (a) $G_{0} / G_{\theta \max }$ as a function of the loading phase angle $\psi=\operatorname{atan}\left(K_{I I} / K_{I}\right)$ for the single homogeneous material by Eq. (12) against that from He et al. (1991). (b) The kinking angle predicted by Eq. (13) against the results from Hutchinson and Suo (1992).

\section{Numerical verification}

In the previous section, we presented the strain energy release rate when a hydraulic crack may propagate in arbitrary directions. We also deduced the critical conditions when the hydraulic crack deflects along a particular path. In this section, we compare the energy release rate ratio expression and the predicted kinking angle against the numerical results from He et al. (1991) and Hutchinson and Suo (1992). We then take the benchmark crack-deflection problem investigated by He and Hutchinson (1989) and compare our theoretical prediction against that given by the authors for the isotropic media case. Finite element (FE) simulations are carried out to examine the applicability of our fracking theory for more complicated cases when there are arbitrarily oriented pre-existing weak zones.

\subsection{Comparison with existing theory}

He et al. (1991) and Hutchinson and Suo (1992) gave a thorough theoretical analysis regarding crack-kink in materials with heterogeneous fracture toughness. The authors also presented numerically results for the critical condition when a straight crack deflects into a particular direction. In Fig. 4a, we show the critical energy release ratio as a function of phase angle $\psi$ from He et al. (1991) and that predicted by Eq. (12). The angle when the kink may occur from Hutchinson and Suo (1992) and that predicted by Eq. (13) is also presented in Fig. 4b. There is perceivable difference between the numerical solutions proposed by He et al. (1991) and Hutchinson and Suo (1992) and the approximate equations we derived by Eq. (3) as $\psi>\pi / 4$. Given the maximum difference is around $7 \%$ at $\psi=\pi / 2$, the Nuismer's equation seems to be a good approximation for the interest of engineering practices for the initiation of crack kinking.

He and Hutchinson (1989) obtained the crack deflection criterion as $\frac{G_{\alpha}}{G_{\theta \max }}=\frac{\left(1-D_{\beta}^{2}\right)\left[\left|a_{2}\right|^{2}+\left|a_{3}\right|^{2}+2 \operatorname{Re}\left(a_{2} a_{3}\right)\right]}{\left(1-D_{\alpha}\right) a_{1}^{2}}$, where $a_{1}$ is a real function in terms of the Dundurs' numbers $D_{\alpha}$ and $D_{\beta}$ and $a_{2}$ and $a_{3}$ are complex functions. They numerically solved a set of integral equations for the functions and then obtained numerical results for $G_{\alpha} / G_{\theta \max }$ in representative geometry. If we let the Dundurs' numbers be zeros, the results could then be used to compare with our theoretical predictions. In Fig. 5a, we show our theoretical prediction (solid line) and the results tabulated by He and Hutchinson (1989) for four typical cases (symbols). Our theoretical results match the predictions from the He-Hutchinson solution of the integral equations. As our closed-form formulae are only applicable to elastically isotropic media, we show the results from He and Hutchinson (1989) when the Dundurs' number $D_{\alpha}$ changes in Fig. 5b. The influence from elastic anisotropy on crack deflection is minor when $D_{\alpha}$ is within the $-0.25-0.1$ range and $D_{\beta}=0$, indicating that our explicit formulae are applicable to media with small elastic anisotropy and significantly anisotropic fracture toughness. Notably, for the special case when $D_{\alpha}=D_{\beta}=0$, the solutions given by He and Hutchinson (1989) can be simplified dramatically in integral form.

\subsection{Verification by FE simulations}

Now, we examine the predictability of our theory for more complicated boundary-value problems. To understand the influence of crustal stresses and crack-surface friction in hydraulic fracture problems, we consider a plate with a weak zone. The stress boundaries and the pressure on the crack surfaces for the FE simulations are shown in Fig. 6. To check whether the theory is sensitive to the tip geometry, we consider two types of crack tips: a sharp crack tip at the junction where the primary crack meets the weak zone (case 'A' at the top right in Fig. 6) and a round tip (case 'B' at the bottom right in 


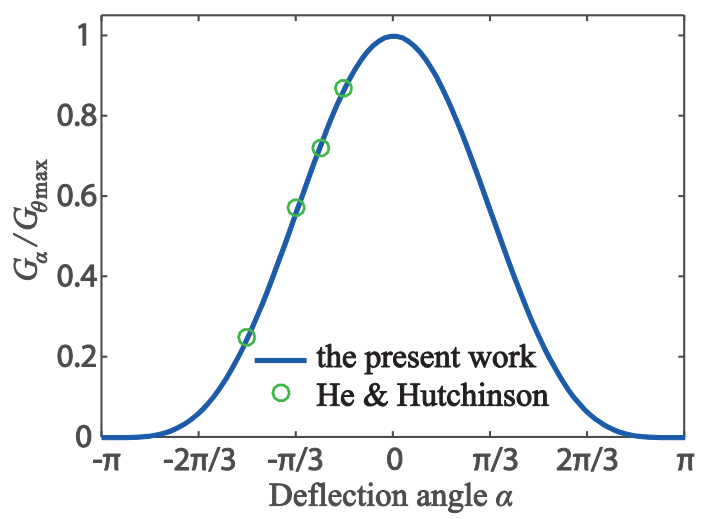

(a)

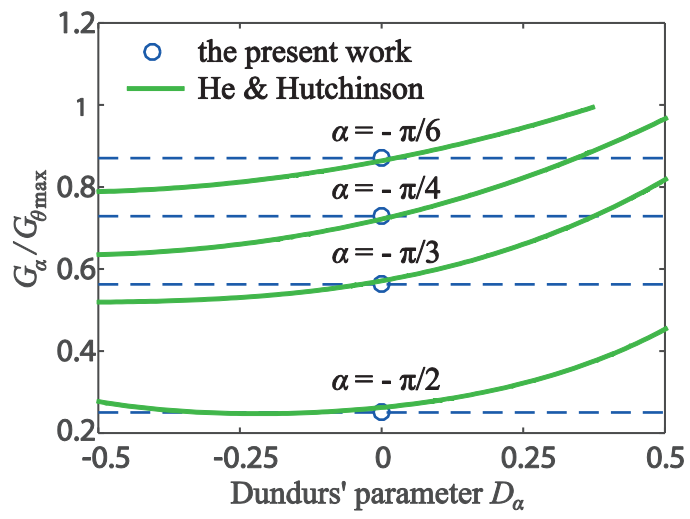

(b)

Fig. 5. Comparison between our theoretical prediction by Eq. (23b) and that from the He-Hutchinson theory (1989). (a) $G_{\alpha} / G_{\theta \text { max }}$ as a function of deflection angles for a symmetrically loaded crack. Here, $D_{\alpha}=0$ and $D_{\beta}=0$. (b) The influence of $D_{\alpha}$ on $G_{\alpha} / G_{\theta \max }$ for a symmetrically loaded crack. Here, $D_{\beta}=0$.

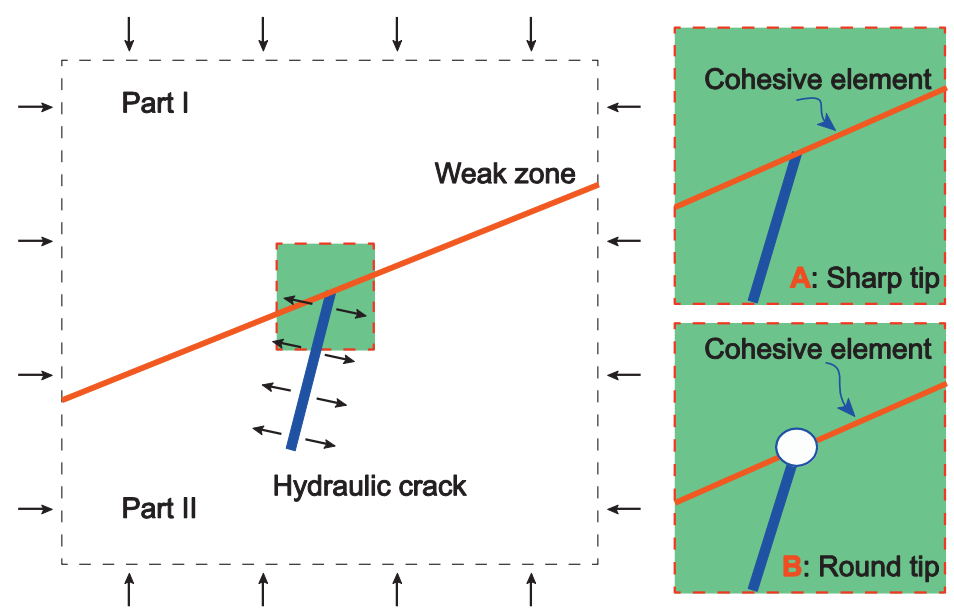

Fig. 6. Finite-element models for the numerical calculation of the strain energy release rate for a hydraulic crack deflecting along a weak zone. Two treatments at the junction between the hydraulic crack and the weak zone are used, as shown at right: the junction could be sharp (top) or round (bottom).

Fig. 6). We calculate the strain energy release rate when the primary crack deflects along the weak zone using the virtual crack closure technique (VCCT) (Rybicki and Kanninen, 1977). The central assumption used in VCCT is that the strain energy released during a crack extension is the same as the energy required to close the crack by the same amount. For more detailed information, refer to Rybicki and Kanninen (1977) and Krueger (2004). We simulate four representative cases and compare the FE results with our theoretical predictions using Eq. (19).

We first consider a symmetrically loaded crack shown in Fig. 7. The crack is under mode I fracture and the strain energy release rate is symmetrical about $\theta=0$. Disregarding the small difference between the FE results by the two models with different crack tip geometry, the energy criterion applied here is insensitive to the exact shape of a crack tip. The FE results agree with our theoretical prediction from Eq. (19b) with $c=1 \mathrm{~m}, \beta=0, \sigma_{h}=35 \mathrm{MPa}, \sigma_{v}=34 \mathrm{MPa}, P=36 \mathrm{MPa}$ and $E^{\prime}=15 \mathrm{GPa}$.

We now consider the influence of crack-plane friction on crack deflection, as illustrated in Fig. 8. Owing to the presence of friction between the crack planes, crack-plane shear stress emerges. For an inclined crack with $\beta=\pi / 3$, the crack is under mode II fracture. The corresponding strain energy release rate $G_{\theta}$ at the crack tip is calculated from FE simulations and Eq. (19a). We consider two situations, $f=0$ and $f=0.2$, in the sample with a central inclined crack with $c=1 \mathrm{~m}$, $\beta=\pi / 3, \sigma_{h}=35 \mathrm{MPa}, \sigma_{v}=34 \mathrm{MPa}, P=34 \mathrm{MPa}$ and $E^{\prime}=15 \mathrm{GPa}$. Fig. 8a shows the FE results against our theoretical prediction by Eq. (19a) with $f=0$. When $f=0.2$, and hence the crack-plane shear stress is non-zero, we see from Fig. $8 \mathrm{~b}$ that the strain energy release rate is much lower than that when $f=0$. This implies that a higher crack-plane friction coefficient gives rise to a lower strain energy release rate and leads to higher critical hydraulic pressure for crack deflection.

For the problem depicted in Fig. 8, we may further increase the hydraulic pressure from $34 \mathrm{MPa}$ to $35 \mathrm{MPa}$ to examine the influence of the hydrostatic pressure on the strain energy release rate. The boundary conditions for the inclined crack 


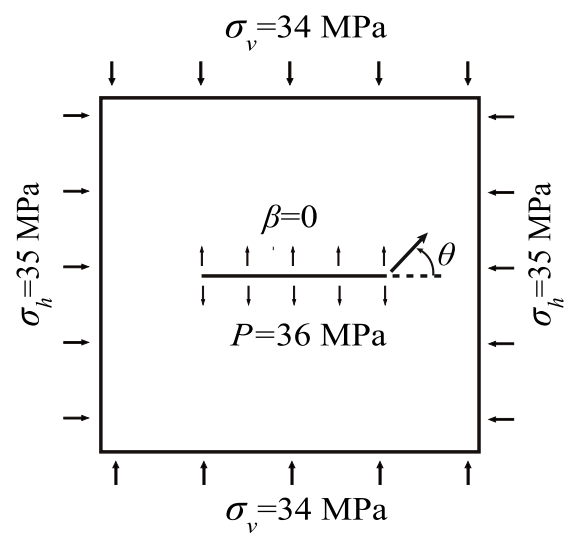

(a)

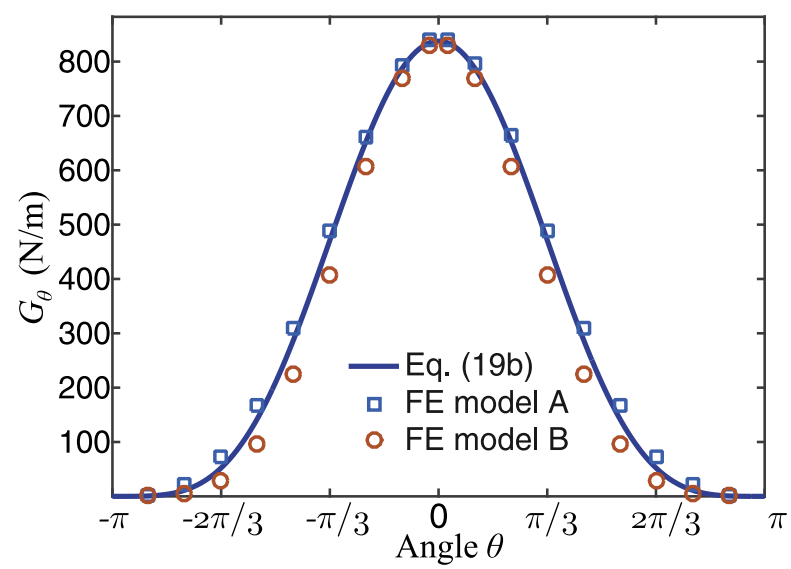

(b)

Fig. 7. Comparison of the FE results and our theoretical prediction for a horizontal crack. (a) The symmetrically loaded crack. (b) The strain energy release rate as a function of the deflection angle from FE simulations and theoretical prediction.

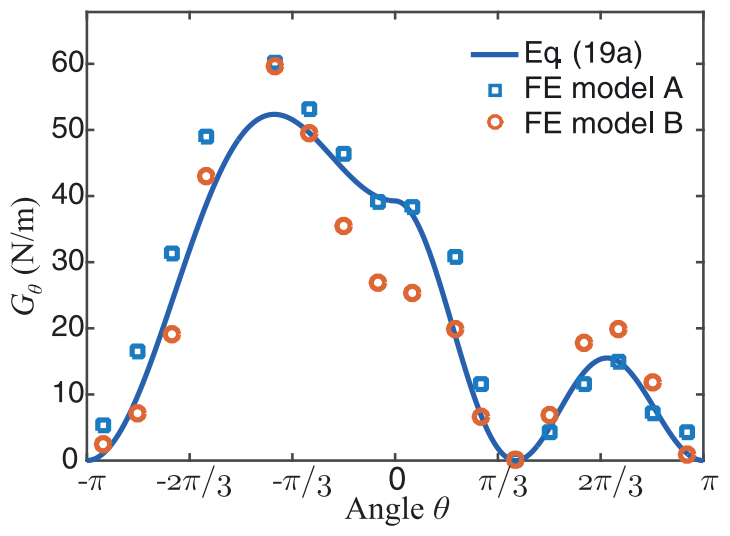

(a)

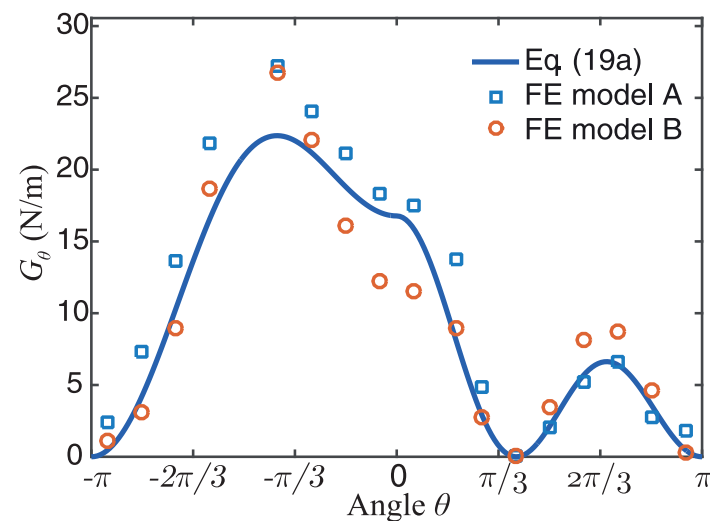

(b)

Fig. 8. Comparison of strain energy release rates from FE results and theoretical predictions for a slanted crack with crack-surface friction: (a) and (b) show the results for friction coefficients $f=0$ and $f=0.2$, respectively.

are shown in Fig. 9a. We show the variation of strain energy release rate with respect to the deflection angle $\theta$ in Fig. $9 \mathrm{~b}$. Notably, the hydrostatic pressure has a significant impact on the energy release rate. From the representative boundaryvalue problems represented in Figs. 7-9, we see that the theoretical predictions using Eq. (19) match the FE simulation results reasonably well.

\section{Application to shale fracking}

With the numerical validation above, we now employ the fracking theoretical deflection criterion to a real hydraulic boundary-value problem and explore the influence of loading environments. We take Mancos shale as the model material. Its fracture toughness of weak discontinuities (along the short-transverse orientation) is approximately 26\% of that of the shale matrix (along the arrester orientation) (Chandler et al., 2016). We assume that the weak bedding planes are horizontal, that its fracture toughness $G_{d c}$ is $20 \mathrm{~N} / \mathrm{m}$ and take a typical effective modulus of the material $E^{\prime}=15 \mathrm{GPa}$ (Chandler et al., 2016). Other parameters of the problem include the crack half-length $c=1 \mathrm{~m}$ and the friction coefficient of the crack surface $f=0.6$. The cracked material is subjected to typical crustal stresses with $\sigma_{h}=50 \mathrm{MPa}$ and $\sigma_{v}=55 \mathrm{MPa}$ (Bažant et al., 2014). We consider different crack impinging angles of $\beta=0, \pi / 6, \pi / 4, \pi / 3$ and $\pi / 2$ and explore the dependence of the strain energy release rate on the hydraulic pressure $P$. Because the condition $\sigma_{v}<\sigma_{h}$ should be satisfied to apply Eqs. (19) and (22), we first perform a transformation (as illustrated in Fig. 10) so that Eqs. (19) and (22) can be directly applied. After the transformation, the equivalent values of parameters $\alpha, \beta, \sigma_{h}$ and $\sigma_{v}$ in the two equations should be replaced by the values of $-\alpha, \pi / 2-\beta, \sigma_{v}$ and $\sigma_{h}$, respectively. 


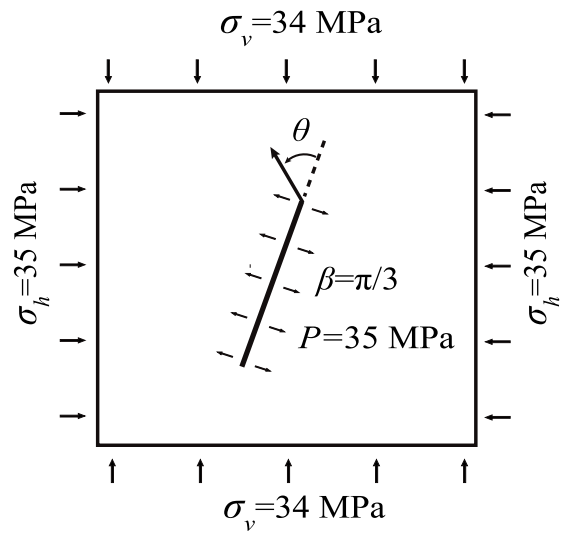

(a)

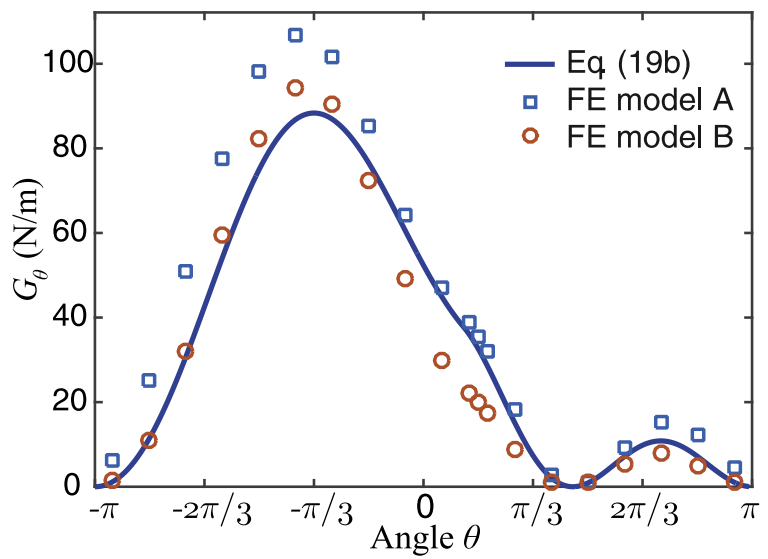

(b)

Fig. 9. The influence of hydrostatic pressure $P$ on crack deflection: FE results and theoretical prediction. (a) The loading conditions for the crack. (b) The strain energy release rate as a function of the deflection angle from FE simulations and the theoretical prediction.

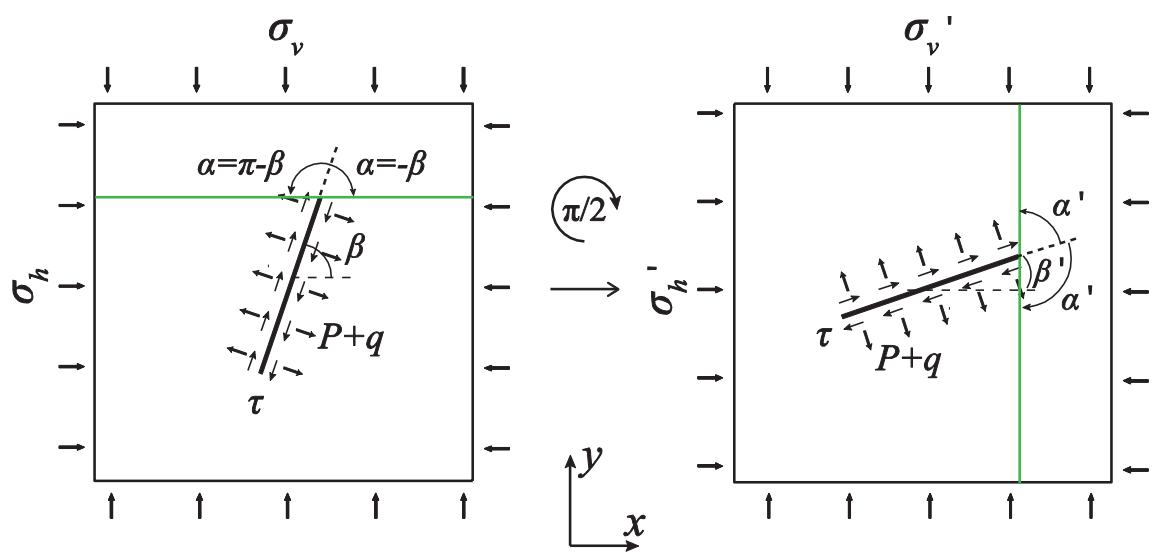

Fig. 10. Model transformation when $\sigma_{v}>\sigma_{h}$. With the transformation, we have $\alpha^{\prime}=-\alpha, \beta^{\prime}=\pi / 2-\beta, \sigma_{h}{ }^{\prime}=\sigma_{v}$ and $\sigma_{v}{ }^{\prime}=\sigma_{h}$. Now, $\left\{\alpha^{\prime}, \beta^{\prime}, \sigma_{h}{ }^{\prime}, \sigma_{v}{ }^{\prime}\right\}$ can be input into Eqs. (19) and (22), then, they can be directly applied.

Under the stress fields, the hydraulic crack can propagate along the two directions of the weak zone, i.e., $\alpha=-\beta$ or $\alpha=\pi-\beta$. With the transformation shown in Fig. 10, we can use Eqs. (19) and (22) directly to obtain the strain energy release rate and the corresponding rate ratio for the Mancos shale cases when the primary crack deflects along the two directions; the results are shown in Fig. 11. For the strain energy release rate shown in Fig. 11a when $\alpha=-\beta$ and for that shown in Fig. 11b when $\alpha=\pi-\beta$, we see that, with increasing hydraulic pressure, the shale may sequentially undergo friction locking (as revealed by Eq. (18a)), mode II fracture (as revealed by Eq. (18b)), and mixed mode fracture (see Eq. (18c)). In the friction locking stage, crack propagation would never happen. In the two later stages, the crack may propagate along the discontinuity once both $G_{\alpha}=G_{d c}=20 \mathrm{~N} / \mathrm{m}$ and $G_{\alpha} / G_{\theta \max }>0.26$ are satisfied. By assuming the crack deflection occurs at the crack tip along the direction $\alpha=-\beta$ and then solving Eq. (19) with $G_{\alpha}=G_{d c}=20 \mathrm{~N} / \mathrm{m}$, we obtain the critical hydraulic pressures to initiate crack deflection for $\beta=0, \pi / 6, \pi / 4, \pi / 3$ and $\pi / 2$, which are 55.31 MPa, $50.81 \mathrm{MPa}$, 49.33 $\mathrm{MPa}$, $50.02 \mathrm{MPa}$ and $50.62 \mathrm{MPa}$, respectively. We see that crack deflection occurs when $\beta=0$ or $\pi / 6$, as $G_{\alpha} / G_{\theta \text { max }}>0.26$ (Fig. 11a). If the crack propagates into the opposite direction of the weak zone, i.e., $\alpha=\pi-\beta$, the critical hydraulic pressures are 51.16 MPa, 49.05 MPa, 48.21 MPa and 50.62 MPa for $\beta=\pi / 6, \pi / 4, \pi / 3$ and $\pi / 2$, respectively. When $\beta=0$, the solution is trivial. It is evident from Fig. 11b that for $\beta=\pi / 4$ or $\pi / 3$, we have $G_{\alpha} / G_{\theta \max }>0.26$ and the hydraulic crack would deflect into the discontinuity. In addition to the four scenarios in which crack deflection occurs, the crack may extend in the matrix along the maximum energy release rate direction $\alpha=\theta_{0}=0$ at $50.61 \mathrm{MPa}$ for $\beta=\pi / 2$, by solving the equality $G_{\theta \max }=G_{m c}=76.92 \mathrm{~N} / \mathrm{m}$. For the four deflection scenarios, we find that the weak plane at the crack junction will experience mode II fracture except in the special case when $\beta=0$. It implies that the hydraulic fracturing in weak planes of shales is mode II type in most cases. This theoretical finding is consistent with experimental observation using microseismic monitoring (Yousefzadeh et al., 2015). It helps to shed light on the so called geomechanical paradox that microseismic data suggest 


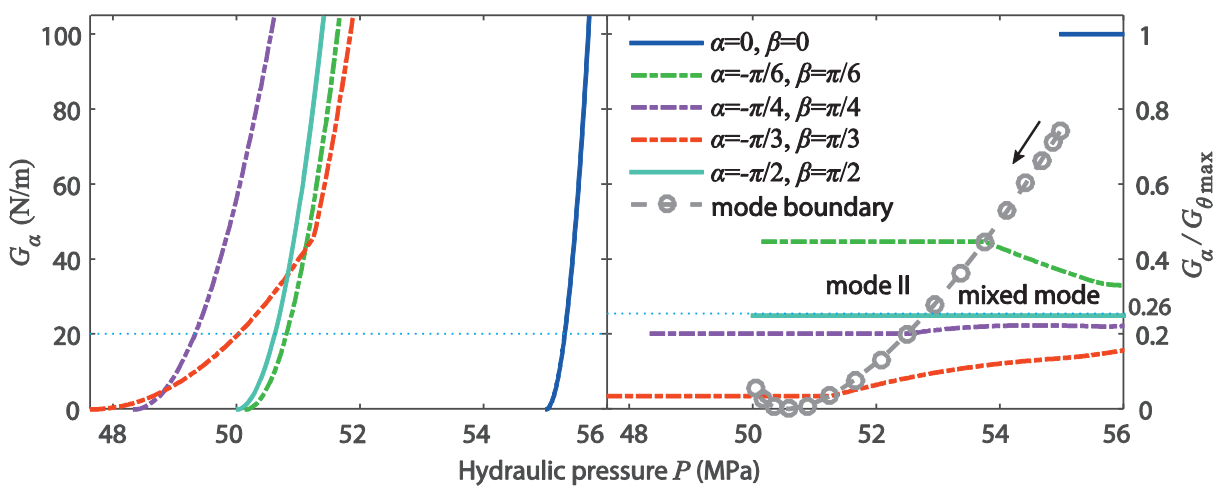

(a)

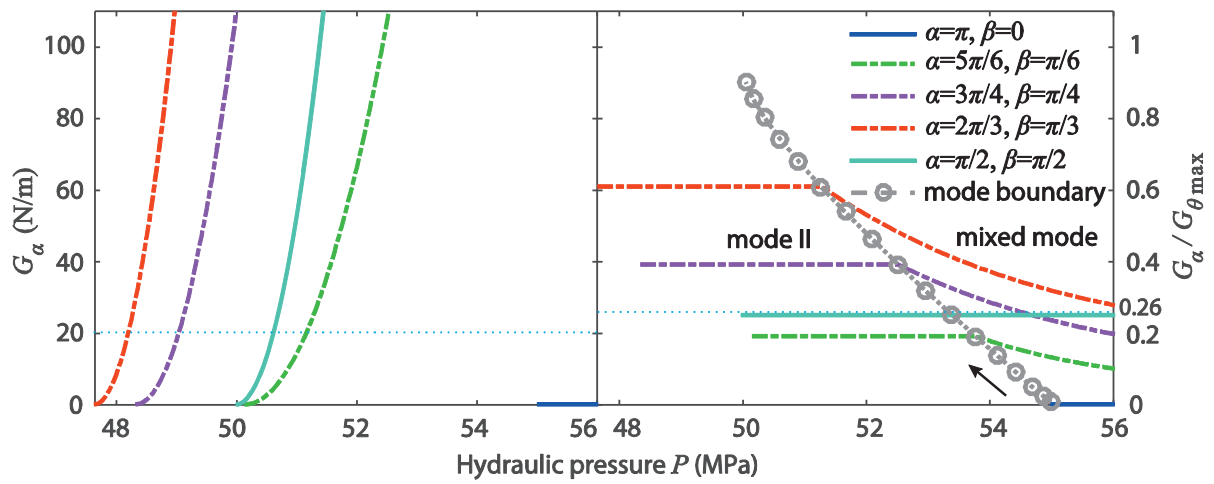

(b)

Fig. 11. Strain energy release rates along the two directions of the weak zone with increasing hydraulic pressure $P$ for Mancos shale. (a) Strain energy release rate and normalized strain energy release rate along the weak zone with $\alpha=-\beta$. (b) Strain energy release rate and normalized strain energy release rate along the weak zone with $\alpha=\pi-\beta$. The mode-transition boundary for different $\beta$ values as the hydraulic pressure $P$ changes is also shown: for each $\beta$ value, the hydraulic crack is mode II fracture in the left pressure range of the curve and is mixed mode fracture in the right pressure range of the curve.

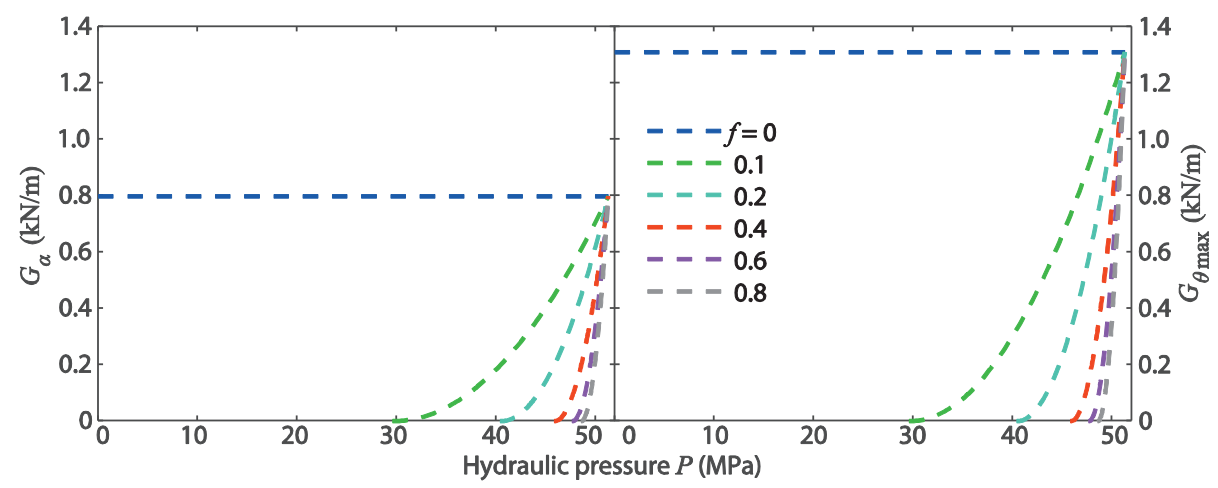

Fig. 12. Influence of the friction coefficient $f$ of crack surfaces on the strain energy release rates for Mancos shale when $\alpha=2 \pi / 3$, $\beta=\pi / 3$. The friction only works in mode II fracture stage.

a strong shearing component for most of microseismic deformation while the classical fracture mechanics views hydraulic fracturing a predominantly tensile deformation phenomenon (Maxwell, 2011).

We illustrate the effects of crack surface friction and anisotropic crustal stresses on hydraulic fracture propagation. Notably, the friction has no influence on the ratio $G_{\alpha} / G_{\theta \max }$ and the isotropic extension angle $\theta_{0}$, namely, no influence on the propagation direction of a crack, as revealed by Eq. (22) and Eqs. (20b) and (21b). The friction only plays a role on the critical propagation loads in mode II fracture stage, as indicated by Eqs. (19a) and (20a). To illustrate the influence of friction, we calculate the strain energy release rates in mode II fracture stage for Mancos shale when $\alpha=2 \pi / 3$ and $\beta=\pi / 3$. Fig. 12 


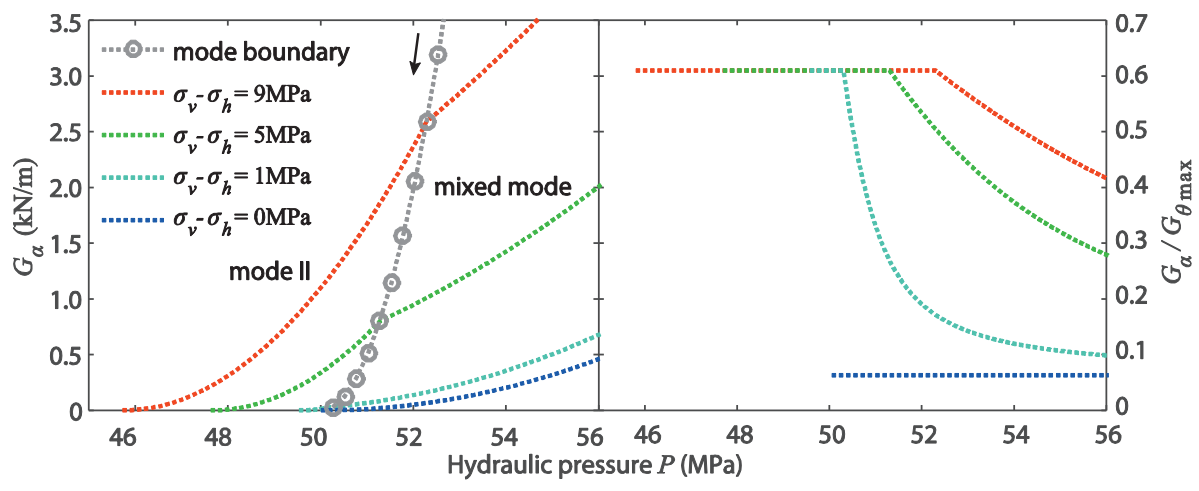

Fig. 13. Strain energy release rate and normalized strain energy release rate as a function of hydraulic pressures for different crustal stress differences, showing the influence of the crustal stress anisotropy on the strain energy release rate for Mancos shale with $\alpha=2 \pi / 3, \beta=\pi / 3$.

presents the strain energy release rates as a function of hydraulic pressures for several typical friction coefficient values. It is clear that cracks prefer to propagate into weak regions or matrices if the crack-plane friction coefficient is lower. Such a conclusion justifies the use of slick-water, which lubricates the crack surfaces and promotes crack propagation (King, 2012).

We now examine how the difference between the horizontal and vertical crustal stresses influences crack propagation. Notably, in mode II fracture stage Eqs. (20b) and (22a) imply that the stress difference has no influence on the propagation direction of a crack and that the impinging angle between a crack and a weak interface is the only determining factor of the deflection direction. In Fig. 13, we show the strain energy release rates of a particularly oriented crack subjected to different combinations of crustal stresses. The results indicate that a greater difference in crustal stresses would lead to lower critical fracturing pressures and retard the transition from mode II to mix-mode fracture and is therefore helpful for shale fracking. This prediction is also consistent with engineering practice (King, 2012) and numerical simulation results (Weng et al., 2011; Guo et al., 2015).

\section{Conclusions}

In this paper, the hydraulic fracture deflection criterion for shale fracking is investigated theoretically. We extend the HeHutchinson theory (1989) for hydraulic crack deflection and give the closed-form formulae of the strain energy release rate of a crack with arbitrary angle with respect to the crustal stress. By discarding the nonphysical term in the conventional strain energy release rate formula (Nuismer, 1975), we deduce the closed-form solution to the hydraulic crack deflection criterion when factors including crack-plane friction and crustal stresses are taken into account. The theoretical results were in agreement with the predictions from He and Hutchinson (1989) and that from FE simulations. In addition, we apply our theory to a real hydraulic fracture problem and obtain the following conclusions:

(1) With increasing hydraulic pressure, a shale formation may sequentially undergo friction locking, mode II fracture, and mixed mode fracture. Type II fracture along the weak interfaces generally dominates the hydraulic fracturing process;

(2) A smaller friction coefficient of crack surfaces leads to lower critical hydraulic pressure to crack the shale matrix or weak regions. Similarly, if the crustal stress difference is greater, the shale is easier to fracture;

(3) In mode II fracture stage, crack-plane friction and crustal stress differences do not influence crack propagation directions and the impinging angle between the hydraulic crack and the weak interfaces is the determining factor that accounts for crack deflection.

As a first-order approximation, the presented fracking theory can be applied to estimate the critical hydraulic pressure for the successful formation of permeable cracking networks in the sedimentary rocks with natural weak zones. Such a process is typically influenced by crack deflection, friction, and crustal stress. In addition to the application of the closedform solutions for the shale hydraulic fracture under the influence of crack-plane friction and crustal stresses, they may be used in fracture analysis for other brittle media with heterogeneous fracture properties.

\section{Acknowledgment}

The authors acknowledge support from the National Natural Science Foundation of China (NSFC) (Grant no. 11425211).

\section{Appendix A}

Here, we show how the strain energy release rate may be written in terms of J-integrals. For an arbitrary counterclockwise path $\Gamma$ around the tip of a crack, Knowles and Sternberg (1972) discovered two related path-independent integrals 
$J_{1}$ and $J_{2}$, where $J_{1}$ is identical to Rice's J-integral (Rice, 1968). According to Eischen (1987), we have:

$$
J_{1}=\left(K_{I}^{2}+K_{I I}^{2}\right) / E^{\prime} \text {, and } J_{2}=-2 K_{I} K_{I I} / E^{\prime} \text {. }
$$

Therefore, we have $J_{1}+J_{2}=\left(K_{I}-K_{I I}\right)^{2} / E^{\prime} \geq 0$ and $J_{1}-J_{2}=\left(K_{I}+K_{I I}\right)^{2} / E^{\prime} \geq 0$. By substituting Eq. (A1) into (5), we derive two sets of J-type formulae for the strain energy release rate $G_{\theta}$ as follows:

$$
\begin{aligned}
& G_{\theta}=\left\{\begin{array}{cl}
\frac{\cos ^{2}\left(\frac{\theta}{2}\right)\left(J_{1}-\bar{J}\right)\left(J_{1}+\bar{J}-3\left(\bar{J}+J_{1}\right) \cos \theta+J_{2} \sin \theta\right)^{2}}{8 J_{2}{ }^{2}}, & \frac{J_{2} \cos \left(\frac{\theta}{2}\right)+3\left(\bar{J}+J_{1}\right) \sin \left(\frac{\theta}{2}\right)}{\sqrt{2} J_{2}}<0 \\
\frac{1}{2} \cos ^{2}\left(\frac{\theta}{2}\right)\left[3 J_{1}+2 \bar{J}-\left(2 \bar{J}+J_{1}\right) \cos \theta+2 J_{2} \sin \theta\right], & \frac{J_{2} \cos \left(\frac{\theta}{2}\right)+3\left(\bar{J}+J_{1}\right) \sin \left(\frac{\theta}{2}\right)}{\sqrt{2} J_{2}} \geq 0
\end{array},\right. \\
& G_{\theta}=\left\{\begin{array}{cc}
\frac{\cos ^{2}\left(\frac{\theta}{2}\right)\left(J_{1}+\bar{J}\right)\left[J_{1}-\bar{J}+3\left(\bar{J}-J_{1}\right) \cos \theta+J_{2} \sin \theta\right]^{2}}{8 J_{2}{ }^{2}}, & \frac{J_{2} \cos \left(\frac{\theta}{2}\right)-3\left(\bar{J}-J_{1}\right) \sin \left(\frac{\theta}{2}\right)}{\sqrt{2} J_{2}}<0 \\
\frac{1}{2} \cos ^{2}\left(\frac{\theta}{2}\right)\left[3 J_{1}-2 \bar{J}+\left(2 \bar{J}-J_{1}\right) \cos \theta+2 J_{2} \sin \theta\right], & \frac{J_{2} \cos \left(\frac{\theta}{2}\right)-3\left(\bar{J}-J_{1}\right) \sin \left(\frac{\theta}{2}\right)}{\sqrt{2} J_{2}} \geq 0
\end{array},\right.
\end{aligned}
$$

where $\bar{J}=\sqrt{J_{1}{ }^{2}-J_{2}{ }^{2}}$. By substituting Eq. (A1) into (7), we obtain the following expression for the maximum strain energy release rate:

$$
G_{\theta \max }=\frac{1}{2} \cos ^{2}\left(\frac{\theta_{0}}{2}\right)\left[3 J_{1}+2 \bar{J}-\left(2 \bar{J}+J_{1}\right) \cos \theta_{0}+2 J_{2} \sin \theta_{0}\right] .
$$
(8):

The corresponding angle $\theta_{0}$ can be determined by the following equation, which is gained by substituting Eq. (A1) into

$$
\theta_{0}=\left\{\begin{array}{ll}
-\operatorname{acos}\left(\frac{3 J_{1}+3 \bar{J}+\sqrt{2 J_{1}{ }^{2}+7 J_{2}{ }^{2}-2 J_{1} \bar{J}}}{10 J_{1}+8 \bar{J}}\right) & \text { for } J_{2}<0 \text { and } J_{1}>-J_{2} \\
\operatorname{acos}\left(\frac{3 J_{1}+3 \bar{J}+\sqrt{2 J_{1}{ }^{2}+7 J_{2}{ }^{2}-2 J_{1} \bar{J}}}{10 J_{1}+8 \bar{J}}\right) & \text { for } J_{2}>0 \text { and } J_{1} \geq J_{2} \\
\pm \operatorname{acos}(1 / 3) \quad \text { when } J_{2}=0
\end{array} .\right.
$$

\section{References}

Anderson, T.L., 2005. Fracture Mechanics: Fundamentals and Applications. CRC Press, p. 84.

Amestoy, M., Leblond, J., 1992. Crack paths in plane situations-II. Detailed form of the expansion of the stress intensity factors. Int. J. Solids Struct. 29, 465-501.

Bažant, Z.P., Salviato, M., Chau, V.T., Visnawathan, H., Zubelewicz, A., 2014. Why fracking works. J. Appl. Mech. 81, 101010.

Bilby, B., Cardew, G., Howard, I., 1977. Stress intensity factors at the tips of kinked and forked cracks. In: Fourth International Conference on Fracture, vol. 3. University of Waterloo Press, pp. 197-200.

Bunger, A., Detournay, E., 2008. Experimental validation of the tip asymptotics for a fluid-driven crack. J. Mech. Phys. Solids 56, 3101-3115.

Bunger, A.P., Gordeliy, E., Detournay, E., 2013. Comparison between laboratory experiments and coupled simulations of saucer-shaped hydraulic fractures in homogeneous brittle-elastic solids. J. Mech. Phys. Solids 61, 1636-1654.

Chandler, M.R., Meredith, P.G., Brantut, N., Crawford, B.R., 2016. Fracture toughness anisotropy in shale. J. Geophys. Res. $121,1706-1729$.

Chuprakov, D., Melchaeva, O., Prioul, R., 2014. Injection-sensitive mechanics of hydraulic fracture interaction with discontinuities. Rock Mech. Rock Eng. 47, 1625-1640.

Cook, T.S., Erdogan, F., 1972. Stresses in bonded materials with a crack perpendicular to the interface. Int. J. Eng. Sci. $10,677-697$.

Cotterell, B., Rice, J., 1980. Slightly curved or kinked cracks. Int. J. Fract. 16, 155-169.

Dahi-Taleghani, A., Olson, J.E., 2011. Numerical modeling of multistranded hydraulic fracture propagation: accounting for the interaction between induced and natural fractures. Soc. Pet. Eng. J. 16, 575-581.

Dahi-Taleghani, A., Olson, J.E., 2014. How natural fractures could affect hydraulic fracture geometry. Soc. Pet. Eng. J. $19,161-171$.

Dundurs, J., 1969. Edge-bonded dissimilar orthogonal elastic wedge. J. Appl. Mech. 36, 650-652.

Eischen, J.W., 1987. An improved method for computing the J2 integral. Eng. Fract. Mech. 26, 691-700.

Erdogan, F., Sih, G.C., 1963. On the crack extension in plates under plane loading and transverse shear. J. Basic Eng. 85, $519-525$.

Gale, J.F., Laubach, S.E., Olson, J.E., Eichhubl, P., Fall, A., 2014. Natural fractures in shale: a review and new observations. AAPG Bull. 98, $2165-2216$.

Gale, J.F., Reed, R.M., Holder, J., 2007. Natural fractures in the Barnett Shale and their importance for hydraulic fracture treatments. AAPG Bull. 91, 603-622.

Grassl, P., Fahy, C., Gallipoli, D., Wheeler, S.J., 2015. On a 2D hydro-mechanical lattice approach for modelling hydraulic fracture. J. Mech. Phys. Solids 75, $104-118$.

Griffith, A.A., 1921. The phenomena of rupture and flow in solids. Philos. Trans. R. Soc. London Ser. A 221, 163-198.

Guo, J., Zhao, X., Zhu, H., Zhang, X., Pan, R., 2015. Numerical simulation of interaction of hydraulic fracture and natural fracture based on the cohesive zone finite element method. J. Nat. Gas Sci. Eng. 25, 180-188.

Gupta, V., Argon, A., Suo, Z., 1992. Crack deflection at an interface between two orthotopic media. J. Appl. Mech. 59, S79-S87.

Hayashi, K., Nemat-Nasser, S., 1981. Energy-release rate and crack kinking under combined loading. J. Appl. Mech. 48, 520-524.

He, M., Bartlett, A.H., Evans, A.G., Hutchinson, J.W., 1991. Kinking of a crack out of an interface: role of in-plane stress. J. Am. Ceram. Soc. 74, 767-771.

He, M., Hutchinson, J.W., 1989. Crack deflection at an interface between dissimilar elastic materials. Int. J. Solids Struct. 25, $1053-1067$.

Hussain, M., Pu, S., Underwood, J., 1973. Strain energy release rate for a crack under combined mode I and mode II. In: Proceedings of the 1973 National Symposium on Fracture Mechanics, vol. 560. ASTM Special Technical Publication, pp. 2-28.

Hutchinson, J.W., Mear, M., Rice, J.R., 1987. Crack paralleling an interface between dissimilar materials. J. Appl. Mech. 54, 828-832. 
Hutchinson, J.W., Suo, Z., 1992. Mixed mode cracking in layered materials. Adv. Appl. Mech. 29, 63-191.

Irwin, G.R., 1957. Analysis of stresses and strains near the end of a crack traversing a plate. J. Appl. Mech. 24, 361-364.

King, G.E., 2012. Hydraulic fracturing 101: what every representative, environmentalist, regulator, reporter, investor, university researcher, neighbor and engineer should know about estimating frac risk and improving frac performance in unconventional gas and oil wells. In: Society of Petroleum Engineers Hydraulic Fracturing Technology Conference. Woodlands, Texas, USA, pp. 1-80.

Knowles, J.K., Sternberg, E., 1972. On a class of conservation laws in linearized and finite elastostatics. Arch. Rat. Mech. Anal. 44, 187-211.

Kresse, O., Weng, X., Gu, H., Wu, R., 2013. Numerical modeling of hydraulic fractures interaction in complex naturally fractured formations. Rock Mech. Rock Eng. 46, 555-568.

Krueger, R., 2004. Virtual crack closure technique: history, approach, and applications. Appl. Mech. Rev. 57, $109-143$.

Lecampion, B., Desroches, J., 2015. Simultaneous initiation and growth of multiple radial hydraulic fractures from a horizontal wellbore. J. Mech. Phys. Solids $82,235-258$.

Lee, H.P., Olson, J.E., Holder, J., Gale, J.F., Myers, R.D., 2015. The interaction of propagating opening mode fractures with preexisting discontinuities in shale. J. Geophys. Res. 120, 169-181.

Lu, M.C., Erdogan, F., 1983. Stress intensity factors in two bonded elastic layers containing cracks perpendicular to and on the interface-I. Analysis. Eng. Fract. Mech. 18, 491-506.

Martínez, D., Gupta, V., 1994. Energy criterion for crack deflection at an interface between two orthotropic media. J. Mech. Phys. Solids 42, $1247-1271$.

Maxwell, S.C., 2011. Microseismic hydraulic fracture imaging: the path toward optimizing shale gas production. Geophysics 30, 340-346.

Mi, Y., Crisfield, M., Davies, G., Hellweg, H., 1998. Progressive delamination using interface elements. J. Composite Mater. 32, $1246-1272$.

Nuismer, R., 1975. An energy release rate criterion for mixed mode fracture. Int. J. Fract. 11, 245-250.

Parmigiani, J.P., Thouless, M.D., 2006. The roles of toughness and cohesive strength on crack deflection at interfaces. J. Mech. Phys. Solids 54, $266-287$.

Rice, J.R., 1968. A path independent integral and the approximate analysis of strain concentration by notches and cracks. J. Appl. Mech. 35, $379-386$.

Rybicki, E.F., Kanninen, M.F., 1977. A finite element calculation of stress intensity factors by a modified crack closure integral. Eng. Fract. Mech. 9, 931-938.

Salvadori, A., Fantoni, F., 2016. Fracture propagation in brittle materials as a standard dissipative process: general theorems and crack tracking algorithms. J. Mech. Phys. Solids 95, 681-696.

Sun, C.T., Jih, C., 1987. On strain energy release rates for interfacial cracks in bi-material media. Eng. Fract. Mech. 28, 13-20.

Suresh, S., Shih, C., 1986. Plastic near-tip fields for branched cracks. Int. J. Fract. 30, 237-259.

Tada, H., Paris, P., Irwin, G., 2000. The Analysis of Cracks Handbook. ASME Press, New York, p. 143.

Vasin, R.N., Wenk, H.R., Kanitpanyacharoen, W., Matthies, S., Wirth, R., 2013. Elastic anisotropy modeling of Kimmeridge shale. J. Geophys. Res. 118, 3931-3956.

Warpinski, N.R., Teufel, L.W., 1987. Influence of geologic discontinuities on hydraulic fracture propagation. J. Pet. Technol. 39, 209-220.

Wei, Y., Gao, H., Bower, A.F., 2009. Numerical simulations of crack deflection at a twist-misoriented grain boundary between two ideally brittle crystals. J. Mech. Phys. Solids 57, 1865-1879.

Weng, X., Kresse, O., Cohen, C., Wu, R., Gu, H., 2011. Modeling of hydraulic-fracture-network propagation in a naturally fractured formation. Soc. Pet. Eng. Prod. Oper. 26, 368-380.

Williams, M.L., 1957. On the stress distribution at the base of a stationary crack. J. Appl. Mech. 24, 109-114.

Yousefzadeh, A., Li, Q., Aguilera, R., 2015. Microseismic 101: Monitoring and evaluating hydraulic fracturing to improve the efficiency of oil and gas recovery from unconventional reservoirs. In: Society of Petroleum Engineers Latin American and Caribbean Petroleum Engineering Conference. Quito, Ecuador, pp. 1-63.

Zak, A.R., Williams, M.L., 1963. Crack point stress singularities at a bi-material interface. J. Appl. Mech. 30, $142-143$.

Zeng, X., Wei, Y., 2016. The influence of crack-orientation distribution on the mechanical properties of pre-cracked brittle media. Int. J. Solids Struct. 96, 64-73.

Zhang, X., Jeffrey, R.G., 2006. The role of friction and secondary flaws on deflection and re-initiation of hydraulic fractures at orthogonal pre-existing fractures. Geophys. J. Int. 166, 1454-1465.

Zhang, X., Jeffrey, R.G., Thiercelin, M., 2007. Deflection and propagation of fluid-driven fractures at frictional bedding interfaces: a numerical investigation. J. Struct. Geol. 29, 396-410.

Zou, Y., Ma, X., Zhang, S., Zhou, T., Li, H., 2016. Numerical investigation into the influence of bedding plane on hydraulic fracture network propagation in shale formations. Rock Mech. Rock Eng. 49, 3597-3614. 